\title{
Analytical Method for Electron Gun Calculation
}

\author{
Oleg D. Potapkin
}

Moscow State Technical University of Radioengineering, Electronics \& Automatics, Prospect

Vernadskogo 78, 119454 Moscow, Russia.

\begin{abstract}
The model of thermionic electron gun was developed. The Dirichlet problem for the cylinder (the Wehnelt electrode) restricted by two bottoms, one of them imitates a plane cathode and another imitates the equipotential surface, was solved analytically. It allows to study electron optical properties of the gun and its behaviour in dependence on Wehnelt potential for different cylinder depths. When the focal distance and the crossover size have the minimal value, this mode is called a work one. The crossover size and the beam half-angle values in this mode were approximated and the analytical method for electron gun calculation was developed.
\end{abstract}

\section{Introduction}

Guns with a plane cathode are widely used in electron beam lithography devices of high throughput [14]. The most known is Lauer's model for thermionic guns with a round cathode tip [5], his other model for the gun with a plane cathode is less known [6]. But this model has the disadvantages. It is difficult to find the emission region size because the uniform field is supposed to be near the plane cathode. As it follows from the results in order to get the minimal crossover size one should have Wehnelt potential value close to the cut-off potential but it contradicts to the experience.

A new semianalytical model called "Equivalent cylinder" has been developed for the thermionic electron gun field [7-9].

The model consists of the cylinder of length $L$ which is restricted by two bottoms of radius $R$, the left one imitates a plane cathode, the right bottom imitates the equipotential surface with $U_{w}{ }^{0}$ potential on it. The potential on the cylinder generatrix is $U_{w}$. The correspondent Dirichlet problem has the two solutions:

$$
U(z, r)=U_{w}{ }^{0} \sum_{\mathrm{n}=1} v_{n}(L / R) M_{n} J_{0}\left(\lambda_{n} r / R\right) \operatorname{sh}\left(\lambda_{n} z / R\right)+U_{w} \sum_{\mathrm{k}=0} w_{k}(L / R) I_{0}\left(\alpha_{k} r / L\right) \sin \left(\alpha_{k} z / L\right),
$$

where $v_{n}(L / R) M_{n}=2 / \lambda_{n} \mathrm{~J}_{1}\left(\lambda_{n}\right) \operatorname{sh}\left(\lambda_{n} L / R\right), w_{k}(L / R)=4 / \pi(2 k+1) \mathrm{I}_{0}((2 k+1) \pi R / L), \alpha_{k}=(2 k+1) \pi, \lambda_{n}$ are the roots of the equation $\mathrm{J}_{0}\left(\lambda_{n}\right)=0$. $\mathrm{J}_{0}\left(\lambda_{n} r / R\right)$ is the Bessel function of the first kind and zero order $(v=0), \mathrm{I}_{0}\left(\alpha_{k} r / L\right)$ is the modified Bessel function of zero order $(v=0)$. The first solution converges badly near the right bottom, so it was approximated. The field between the cylinder and an anode with a hole has the length $L_{0}$ (Figure 1).

Paraxial trajectories can be plotted readily by using Runge-Kutta procedure with a constant step. Nonparaxial trajectories can be plotted by using the same procedure but it requires much more steps of integration. The number of steps can run up to 4000 for the trajectories started with big (more than $45^{\circ}$ ) angles. When the number of steps is insufficient, the outer branches of emittance diagrams have wrong shapes for big $U_{w}{ }^{0}$ values [7].

The space charge influence is supposed to be low. 


\section{Cathode lens parameters}

The field strength at the cathode decreases with the absolute value of $U_{w}$ increasing and the crossover size as well as the back focal distance behave in more complicated manner than it goes on in the Schottky gun [10].

When the potential on the Wehnelt changes, the focal distance of the cathode lens has a minimum on its dependence on Wehnelt potential (Figure 2). This mode is often used in electron beam shaped lithography devices so it can be called a work mode. The real crossover is at the bore plane in this mode. When the cathode lens image plane goes to infinity, one has a semitelefocus mode; the real crossover can be formed in this case. When the asymptotic focal distance goes to infinity, one has a telefocus mode.

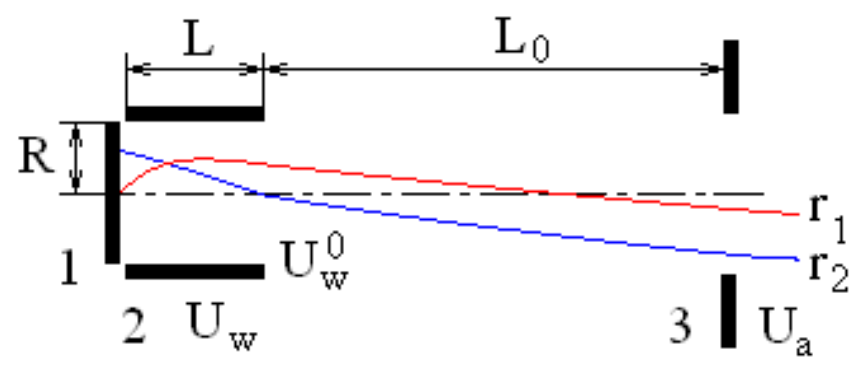

Figure 1. The electron gun model: 1 - cathode, 2 - Wehnelt electrode, 3 - anode, $\mathrm{r}_{1}-$ axial, $\mathrm{r}_{2}-$ field paraxial trajectory.

The work mode is restricted by the semitelefocus mode at low Wehnelt potentials $\left(\left|U_{w}\right|\right)$ and by the telefocus mode at high Wehnelt potentials $\left(\left|U_{w}\right|\right)$ when the bias is close to the correspondent cut-off value $U_{c}$.

(a)

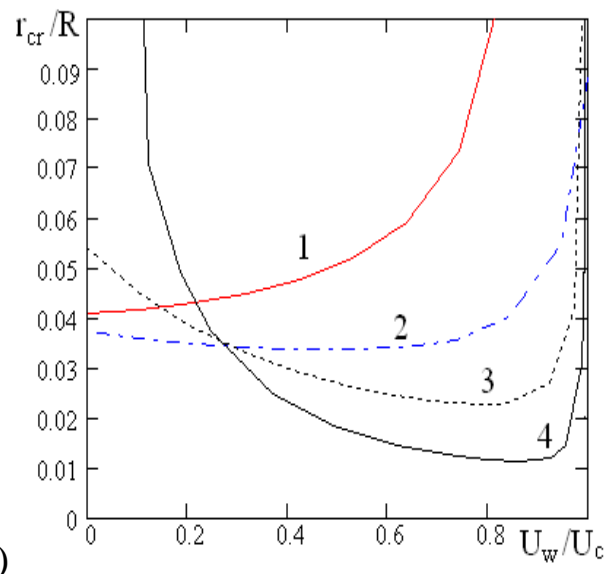

(b)

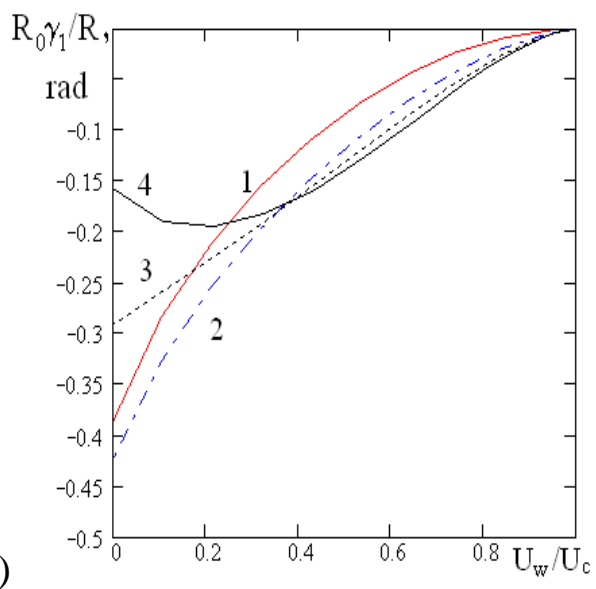

Figure 2. Electron gun properties in dependence on reduced Wehnelt potential: (a) the reduced crossover size $r_{c r} / R$, (b) the half-angle $R_{0} \gamma_{1} / R ; 1: L / R=2,2: L / R=1.5,3: L / R=1,4: L / R=0.75 ; U_{w}{ }^{0}=$ $1 \mathrm{kV}$.

The semitelefocus mode takes place for the potential value

$$
U_{s}=\left(0.68-0.38(L / R)^{2}\right) U_{c}
$$

and when $0.75 \leq L / R \leq 2, U_{w}{ }^{0}=1 \mathrm{kV}$. 
The telefocus mode takes place for the potential value

$$
U_{t}=\left(1.01-0.015(L / R)^{2}\right) U_{c}
$$

and when $0.75 \leq L / R \leq 2, U_{w}{ }^{0}=1 \mathrm{kV}$.

The expressions (2) and (3) are empirical ones, they were found from paraxial trajectory calculation for the electron start energy $u=0.25 \mathrm{eV}$. For other $U_{w}{ }^{0}$ values they give only qualitative dependence of the correspondent potentials on $L / R$ ratio. Moreover, the dependence (3) can change drastically for other start energy values.

(a)

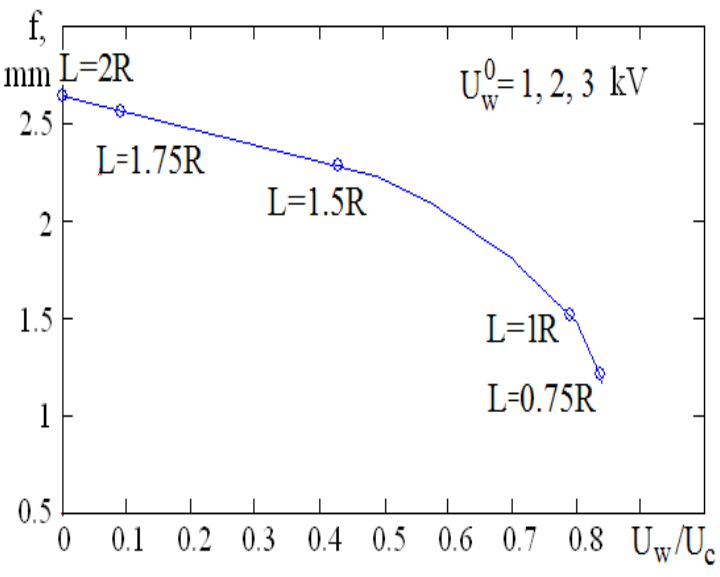

(c)

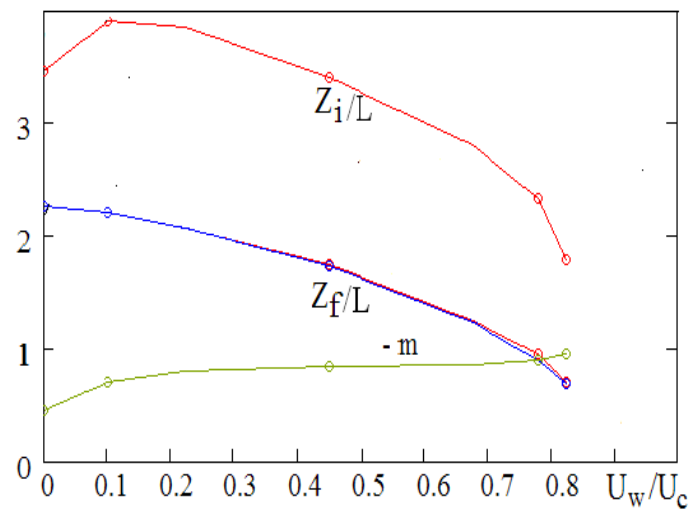

(b)

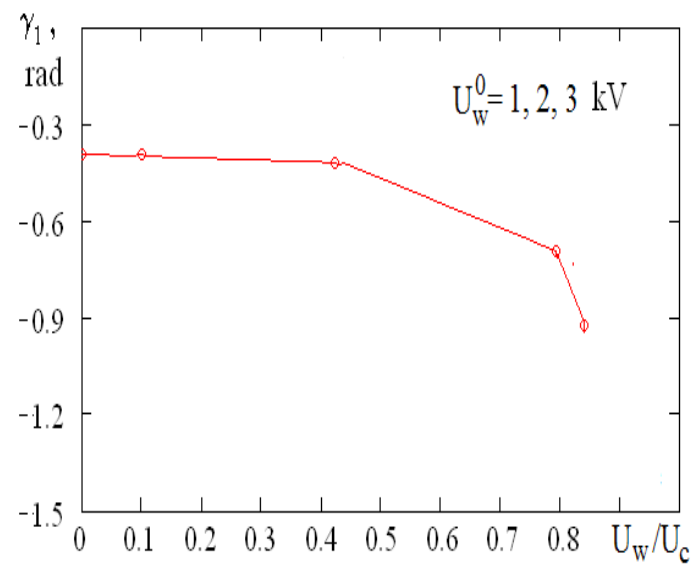

(d)

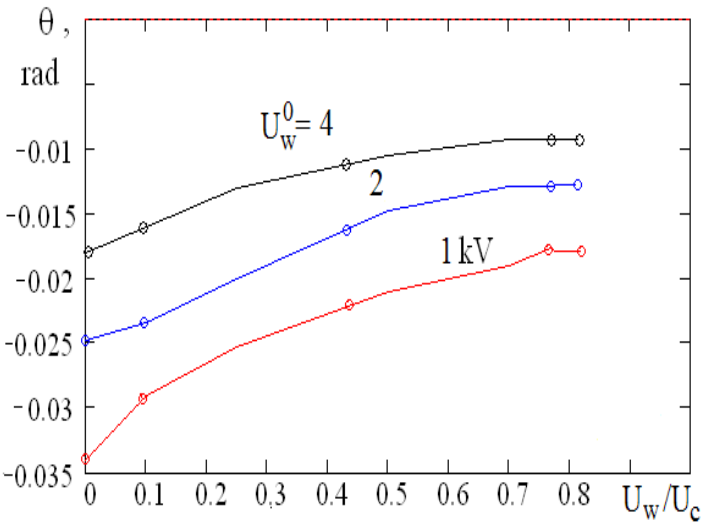

Figure 3. Generalized cathode lens properties: (a) the focal distance dependence on Wehnelt potential, (b) the half-angle dependence on Wehnelt potential, (c) the axial coordinate of the focal plane $Z_{f}$, image plane $Z_{i}$ and the linear magnification $\mathrm{m}$ dependence on Wehnelt potential, (d) the angle of convergence $\theta$ dependence on Wehnelt potential.

It's interesting that the work mode takes place at the same ratio $U_{w p} / U_{c}$ for different $U_{w}{ }^{0}$ :

$$
U_{w p}=\left(0.875-0.38(L / R-0.5)^{2}\right) U_{c} \text {. }
$$

It was found that the minimal focal distance of the cathode lens doesn't depend on the potential at the bore $U_{w}{ }^{0}$. It can be seen in Figure 3. Therefore, the minimal focal distance in the work mode can be approximated by the expression

$$
f / R=2.64-1.8\left(U_{w p} / U_{c}\right)^{2}=2.64-1.8\left(\left(0.875-0.38(L / R-0.5)^{2}\right)\right)^{2} .
$$


The crossover radius (Figures 2 and $3 a$ ) can be written as

$$
r_{c}=f\left(u / U_{w}{ }^{0}\right)^{1 / 2}
$$

where $u=k T / e, k$ - Boltzmann's constant, $T$ - cathode temperature, $e$ - electron charge. The cathode lens half-angle (Figure $2 \mathrm{~b}$ ) can be present in such a way

$$
\gamma=R_{0} / R f=\gamma_{1} R_{0} / R
$$

where $\gamma_{1}$-tangent of $r_{2}$ trajectory angle at the bore plane (Figure $3 \mathrm{~b}$ ), $R_{0}$ - emission region radius:

$$
R_{0} \approx\left(1-U_{w p} / U_{c}\right) R=\left(0.125+0.38(L / R-0.5)^{2}\right) R
$$

$\theta$ is tangent of $r_{1}$ trajectory angle at the bore plane (Figure $3 \mathrm{c}$ ), its values in the work mode are shown in Figure $3 \mathrm{~b}$ for $u=0.25 \mathrm{eV}$.

\section{Influence of the anode space}

The crossover size of the whole gun equals to

$$
r_{c r}^{a}=M^{a} r_{c r}
$$

$M^{a}$ - the linear magnification of the anode space. It is supposed that the real crossover is in the bore.

The half-angle of the whole gun equals to

$$
\gamma^{a}=m^{a} \gamma
$$

$m^{a}$ - the angular magnification of the anode space.

The potential in the bore is

$$
U_{w}{ }^{0}=U_{a} R / \pi L_{0}+U_{w p} .
$$

Up to this point the ratio $U_{w p} / U_{c}$ has been used and it hasn't needed to calculate $U_{w p}$ or $U_{c}$. Meantime the expression (11) requires $U_{w p}$ and $U_{c}$ values. The expression for the cut-off potential was got in the work [9]:

$$
U_{c}=-\frac{0.216 U_{w}^{0}\left(\frac{L}{R}\right)^{\frac{3}{2}} \exp \left(\frac{\pi R}{L}\right)}{\sinh \left(\frac{2.4 L}{R}\right)}=-0.432 U_{w}^{0}\left(\frac{L}{R}\right)^{\frac{3}{2}}\left\{\exp \left(\frac{2.4 L}{R}-\frac{\pi R}{L}\right)-\exp \left(-\frac{2.4 L}{R}-\frac{\pi R}{L}\right)\right\}
$$

\section{Analytical method of calculation}

Let's introduce the notations

$$
x=L / R, y=\pi L_{0} / R
$$

and let's introduce the scale factor

$$
R_{m}=R \text {. }
$$

The scale factor shows that the field trajectory coordinates increase $R_{m}$ times when geometrical sizes increase $R_{m}$ times.

The expression for the crossover size is defined by the following factors: the anode hole factor (the linear magnification $M^{a}=2 y^{1 / 2} /\left(3 y^{1 / 2}-1\right)$ ) [11], the anode space factor $0.9 \leq M^{u} \leq 2$, the factor of the total focal distance $f_{g}$ of the whole gun, the immersion factor $\left(U_{w}{ }^{0} / e U_{a}\right)^{1 / 2}$, the scale factor $R_{m}$. Then the crossover size expression can be written as 


$$
\begin{aligned}
r_{c r}^{a}=\frac{2 y}{3 y^{1 / 2}-1} & M^{u} f_{g}\left(\frac{k T}{e U_{a}}\right)^{1 / 2} R_{m} \\
& =\frac{2 y}{3 y^{1 / 2}-1} M^{u}\left\{2.64-[1.18-0.5(x-0.5)]^{2}\right\}\left(\frac{\pi L_{0}}{R}\right)^{1 / 2}\left(\frac{k T}{e U_{a}}\right)^{1 / 2} R_{m}
\end{aligned}
$$

The half-angle of the whole gun can be represented as the product of angular magnification of the anode hole, the angular magnification of the anode space, the magnitude that is inversely proportional to the focal distance, the emission radius $R_{0}$ and the magnitude that is inversely proportional to the scale factor, in result, one has

$$
\gamma^{a}=\left\{\left(3 y^{1 / 2}-1\right) / 2 y\right\}\left(1 / M^{u}\right)\left\{2.64-[1.18-0.38(x-0.5)]^{2}\right\}^{-1}\left(R / \pi L_{0}\right)^{1 / 2}\left(0.12+0.38(x-0.5)^{2}\right) .
$$

The axial trajectory angle is found from the product of the angle $\theta$ in the real crossover plane and the angular magnification $m^{a}=\left(1 / M^{u}\right)\left(3 y^{1 / 2}-1\right) / 2 y[11]$. Then

$$
\theta^{a}=m^{a} \theta=m^{a}\left\{k T / e U_{w}{ }^{0}\right\}^{1 / 2} / m=\left\{\left(3 y^{1 / 2}-1\right) / 2 y^{1 / 2}\right\}\left(1 / M^{u}\right)\left\{k T / e U_{a}\right\}^{1 / 2} / m,
$$

where $m$ is the cathode lens magnification [5]. The beam angle $\theta^{a}$ doesn't depend on the scale factor. But the linear magnification of the cathode lens depends on the initial energy $k T$, this is the property of a cathode lens. So (17) approximates the result of the semianalytical calculation only for $k T / e=0.25 \mathrm{eV}$ and for $150 \mathrm{~V} \leq U_{w}{ }^{0} \leq 2500 \mathrm{~V}$.

The maximal current density at the gun exit equals

$$
j_{0}=j_{c} /\left(M^{a} M^{u} m\right)^{2}=j_{c} /\left\{2 y^{1.2} /\left(3 y^{1 / 2}-1\right)\right\}^{2}\{1.5-0.5 x\}^{2},
$$

where $j_{c}$ - the cathode current density which is described by Richardson-Dushman formula, $m$ - the cathode lens linear magnification in the work mode.

The beam generator emittance is defined as the product of the crossover diameter and the total angle of the gun:

$$
\varepsilon=4 r_{c r}{ }^{a} \gamma^{a}=4\left(0.12+0.38(x-0.5)^{2}\right)\left\{k T / e U_{a}\right\}^{1 / 2} .
$$

So the emittance is proportional to the emission region reduced size.

The asymptotic crossover plane position is

$$
Z_{F}^{a} \approx L_{0}\left(1-4 y /\left(3 y^{1 / 2}-1\right)\left(y^{1 / 2}+1\right)\right)
$$

for the real crossover displaced in the bore. The asymptotic crossover plane position is measured from the cathode.

\section{Conclusions}

It was shown that the gun asymptotic focal distance is minimal when the real crossover is in the Wehnelt bore. The real focal distance of the cathode lens for this case doesn't depend on the potential in the bore. It allowed to develop the analytical method of the electron gun.

The results showed a good accordance to the data got from home-made program package written on the basis of Charge Density Method [12] but there are two points. The first point revealed that formula (12) gives higher values of cut-off potential than it takes place in reality. The second point revealed that the anode space could have its linear magnification $M^{u} \approx 2$. 
At which extent (different $\left.U_{w}{ }^{0}, L\right)$ space charge has influence can be a subject for future work. The formulae presented here can provide a researcher with the boundary values of the crossover parameters for zero space charge case.

In real electron optical lithography systems aberration of the last lens defines the gun emittance. For high emission current values the most important among these aberrations are Boersh-Loeffler ones [13], they often impose top limit on the emission current (10-100 microamperes as, for instance, in projection lithography from [3]) and on the brightness [4]. But some researchers ignore Boersh-Loeffler aberrations in its work as it was done in development of Variable Shaped Lithography [1] where emission current can reach 300 microamperes. Such cases certainly demand the usage of numerical calculations.

The model can serve as a good benchmark for numerical calculations.

\section{References:}

[1] K Saito et al, Proc. SPIE 2858 (1996), p. 22.

[2] HC Pfeiffer and W Stickel, Proc. SPIE 7378 (2009), 737802.

[3] W DeVore and SD Berger, J. Vac. Sci. Technol. B 14 (1996), p. 3764.

[4] P Petric et al. J. Vac. Sci. Technol. B. 27 (2009), p. 161.

[5] R Lauer, Adv. Opt. Electron Microsc. 8 (1982), p. 137.

[6] R Lauer, Z. Naturforsch. 23a (1968), p. 100.

[7] OD Potapkin, Phys. Procedia 1 (2008), p. 77.

[8] OD Potapkin, Izv. RAN Ser. Fiz. 68 (2004), p. 1362 (in Russian).

[9] OD Potapkin, Bull. RAN Phys. 73 (2009), p. 478.

[10] HH Rose in „Geometrical Charged Particle Optics“, (Springer, Berlin, 2009) p. 349.

[11] M Lenc and I. Müllerová, Ultramicroscopy 41 (1992), p. 411.

[12] PW Hawkes and E Kasper in „Principles of Electron Optics“, (Academic, London, 1995).

[13] P Kruit and H Jansen in "Handbook of Charged Particle Optics", ed. J Orloff, (CRC Press, Boca

Raton, 1997) p.275. 